\title{
Study protocol: improving the transition of care from a non-network hospital back to the patient's medical home
}

Roman A. Ayele ${ }^{1,2^{*}}$ D, Emily Lawrence ${ }^{1}$, Marina McCreight ${ }^{1}$, Kelty Fehling ${ }^{1}$, Jamie Peterson ${ }^{1}$, Russell E. Glasgow ${ }^{4}$, Borsika A. Rabin ${ }^{3,4}$, Robert Burke ${ }^{1,2}$ and Catherine Battaglia ${ }^{1,2}$

\begin{abstract}
Background: The process of transitioning Veterans to primary care following a non-Veterans Affairs (VA) hospitalization can be challenging. Poor transitions result in medical complications and increased hospital readmissions. The goal of this transition of care quality improvement (QI) project is to identify gaps in the current transition process and implement an intervention that bridges the gap and improves the current transition of care process within the Eastern Colorado Health Care System (ECHCS).

Methods: We will employ qualitative methods to understand the current transition of care process back to VA primary care for Veterans who received care in a non-VA hospital in ECHCS. We will conduct in-depth semi-structured interviews with Veterans hospitalized in 2015 in non-VA hospitals as well as both VA and non-VA providers, staff, and administrators involved in the current care transition process. Participants will be recruited using convenience and snowball sampling. Qualitative data analysis will be guided by conventional content analysis and Lean Six Sigma process improvement tools. We will use VA claim data to identify the top ten non-VA hospitals serving rural and urban Veterans by volume and Veterans that received inpatient services at non-VA hospitals.

Informed by both qualitative and quantitative data, we will then develop a transitions care coordinator led intervention to improve the transitions process. We will test the transition of care coordinator intervention using repeated improvement cycles incorporating salient factors in value stream mapping that are important for an efficient and effective transition process. Furthermore, we will complete a value stream map of the transition process at two other VA Medical Centers and test whether an implementation strategy of audit and feedback (the value stream map of the current transition process with the Transition of Care Dashboard) versus audit and feedback with Transition Nurse facilitation of the process using the Resource Guide and Transition of Care Dashboard improves the transition process, continuity of care, patient satisfaction and clinical outcomes.
\end{abstract}

Discussion: Our current transition of care process has shortcomings. An intervention utilizing a transition care coordinator has the potential to improve this process. Transitioning Veterans to primary care following a non-VA hospitalization is a crucial step for improving care coordination for Veterans

\footnotetext{
* Correspondence: roman.ayele@va.gov

${ }^{1}$ Department of Veterans Affairs, Eastern Colorado Health Care System, 1055

Clermont Street, Research (A151), Denver, CO 80220, USA

2University of Colorado, Anschutz Medical Campus, Aurora, CO, USA

Full list of author information is available at the end of the article
}

\section{Ciomed Central}

(c) The Author(s). 2017 Open Access This article is distributed under the terms of the Creative Commons Attribution 4.0 International License (http://creativecommons.org/licenses/by/4.0/), which permits unrestricted use, distribution, and reproduction in any medium, provided you give appropriate credit to the original author(s) and the source, provide a link to the Creative Commons license, and indicate if changes were made. The Creative Commons Public Domain Dedication waiver (http://creativecommons.org/publicdomain/zero/1.0/) applies to the data made available in this article, unless otherwise stated. 


\section{Background}

Care coordination can be highly problematic for health systems to re-establish care with the patient's medical home when patients transition across multiple systems. Deficient care coordination processes often results in adverse clinical events due to fragmented care, lack of communication between providers and patient confusion regarding postdischarge medications and timing of follow-up care [1-5]. In 2011, poorly managed transitions were estimated to cost between $\$ 25$ to $\$ 45$ billion due to avoidable complications and unnecessary hospital readmissions [6]. Transitions are also vulnerable exchange points for patients and caregivers, especially for older patients coping with multiple comorbidities and complex regimens. Patients have identified coordination of care as one of the factors that influences their perception of quality [7]. Care coordination tools such as clinical pathways, information systems, case management, as well as high-quality communication and strong relationships among health care providers need to be available to provide efficient clinical outcomes, and the exchange of critical information among providers [7].

There are several reasons for poor care coordination in the transition from hospital back to primary care. First, different electronic medical record systems make it difficult to transmit medical information between hospitals and physician practices. Consequently, the primary care providers lack information about their patients' hospitalizations [8, 9]. A study in 2007 found that only $12-34 \%$ of primary care providers had received a hospital discharge summary about their patients' hospitalization and had it on hand during their patient's follow-up appointment. Even when discharge summaries are received, they often lack key information, such as test results, treatment course, discharge medications, and follow-up plans further contributing to ineffective care coordination [8].

Providing safe and comprehensive care coordination to Veterans who access care across multiple systems is challenging for the Department of Veterans Affairs (VA). Problems found in other health systems are not uncommon when Veterans are hospitalized in a non-VA hospital and transition back to VA primary care for follow-up. Care coordination tools that ensure a smooth transition are not consistently available. Given that VA hospitals tend to be located in urban areas, Veterans who live further from a VA facility are less likely to rely on the VA for inpatient care $[9,10]$. This increases the Veteran's chance of receiving care at a non-VA hospital. Delivering comprehensive quality health care for these Veterans is often dependent upon the coordination and integration of VA health care services with non-VA care systems.

\section{Objective}

The goal of this transition of care quality improvement (QI) project is to identify gaps in the current transition process and implement an intervention that bridges the gap and improves the current transition of care process within the Eastern Colorado Health Care System (ECHCS). We will use value stream mapping (a Lean Six Sigma tool) to explore the current steps a patient must take to transition from non-VA hospital to VA primary care [11]. Moreover, we will identify inefficiencies in the current process, implement an intervention to provide effective, coordinated care in a team-based environment, and test the effectiveness and sustainability of the intervention.

\section{Conceptual framework and theoretical foundation}

A comprehensive conceptual model called a Practical, Robust Implementation and Sustainability Model (PRISM) will be used for integrating research findings into practice. Rooted in concepts from QI, the Chronic Care Model, the Diffusion of Innovations theory, and measures of population-based effectiveness of translation, PRISM evaluates how a healthcare program or intervention interacts with the recipients to influence program reach, adoption, implementation, maintenance, and effectiveness [12]. Main domains of PRISM include Organizational characteristics, Patient/provider characteristics, Intervention from the perspective of the organization and patient, external environment, and sustainability infrastructure.

PRISM (Fig. 1) PRISM highlights four components that influence implementation success: 1) organizational and participants characteristics; 2) intervention characteristics from the organizational (medical facility) and participants' perspectives (i.e., patients and providers); 3) implementation and sustainability infrastructure (training and support); and 4) external environment. PRISM also identifies a set of important outcomes from the REAIM model (i.e., Reach and Effectiveness, Adoption,

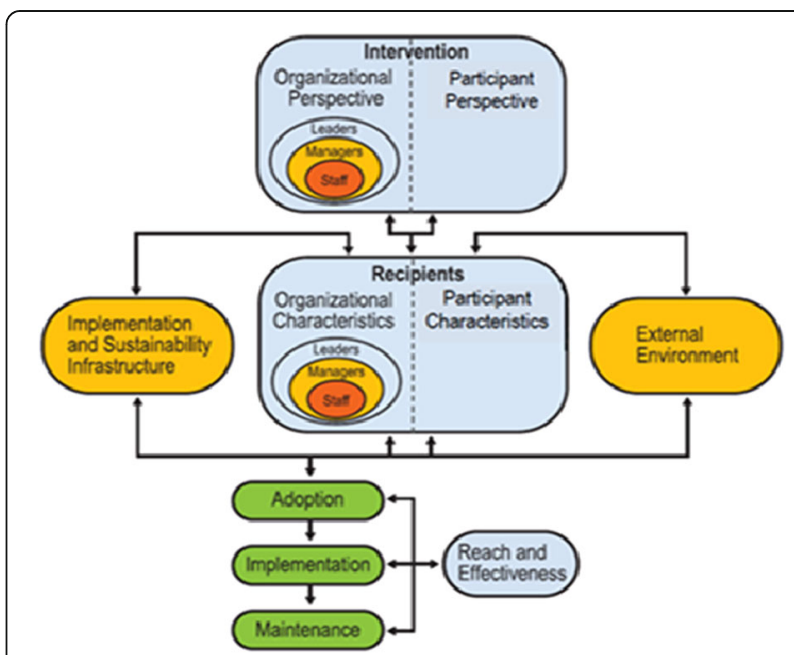

Fig. 1 The PRISM Framework used to guide planning, implementation, and evaluation of the QI project and to frame the implementation core 
Implementation, and Maintenance) for evaluation. Important elements to improve program implementation based on PRISM include creating an environment for encouraging spread, sharing best practices, observing results and adjusting processes accordingly, facilitating use of the intervention, and ensuring adaptability of protocols. These elements will be used in a formative manner and incorporated into our planning and implementation process. Key outcomes and analyses related to the program and implementation strategy will be evaluated using the Reach, Effectiveness, Adoption, Implementation and Maintenance (RE-AIM) measures [13].

We will also use Haggerty's Continuity of Care $(\mathrm{CoC})$ Framework [14] and the principles established in the Transitions of Care Consensus Policy Statement [15], to define an ideal transition of care process. The ideal transitions of care process will address the domains referenced in Table 1 that are associated with improved care transitions [16]. These ideal transitions of care domains will help us guide our intervention to improve the current process and to make it ideal.

\section{Methods and design}

\section{Project description and study design}

This project has three phases. During the first phase (Aim 1) we will: 1) interview key VA providers and staff informants and Veterans hospitalized in a non-VA hospital in 2015 and 2) interview non-VA providers and staff informants from high volume urban and rural hospitals used by ECHCS Veterans. The goals of Aim 1 are to: 1) create a value stream map with current processes and develop an ideal transition of care process, 2) identify gaps in transition of care process between current and ideal processes of care, and 3) create a Transition of
Care Resource Guide for VA and non-VA providers as well as patients that would allow for monitoring and feedback of the process subsequent phases to ensure optimal continuity of care. Phase 1 will be completed during the first year of the project.

During the second phase (Aim 2) we will: 1) develop and pilot test an intervention utilizing a Transition Nurse role and system changes to facilitate continuity of care for Veterans returning to VA primary care at ECHCS after non-VA hospitalization. The goal of Aim 2 is to test the intervention using repeated improvement cycles incorporating factors that are identified as valueadded based on data collected of identified process measures. These data will be monitored and displayed on the Transition of Care Dashboard. We will have both process and outcomes measures such as number of nonVA hospital discharge summaries received within 14 days, number of post discharge follow up visits with VA primary care provider within 14 days of discharge, ER utilization rates 30 days after hospital discharge and re-hospitalization rates 30 days after hospital discharge. Phase 2 will be completed during the second year of the project.

During the third and final phase (Aim 3) we will: 1) complete a value stream map of the transition process at two other VA Medical Centers in Veterans Integrated Service Networks (VISNs) 19 in consultation with the VISN 19 Chief Medical Officer and 2) test whether an implementation strategy of audit and feedback (the value stream map of the current transition process with the Transition of Care Dashboard) versus audit and feedback $[17,18]$ with Transition Nurse facilitation of the process using the Resource Guide and Transition of Care Dashboard improves the transition process, continuity of care, patient satisfaction and clinical outcomes.

Table 1 Key components of an ideal transition in care

\begin{tabular}{|c|c|}
\hline Domains & Description \\
\hline Discharge Planning & $\begin{array}{l}\text { Involves the important principle of planning ahead for hospital discharge while the patient is still being } \\
\text { treated in the hospital. }\end{array}$ \\
\hline Complete communication of information & $\begin{array}{l}\text { Refers to the content that should be included in discharge summaries and other means of information } \\
\text { transfer from hospital to post-discharge care. }\end{array}$ \\
\hline $\begin{array}{l}\text { Availability, timeliness, clarity and } \\
\text { organization of information }\end{array}$ & $\begin{array}{l}\text { Important because post-discharge providers must be able to access and quickly understand the information } \\
\text { they have been provided before assuming care of the patient. }\end{array}$ \\
\hline Medication Safety & This is of central importance because medications are responsible for most post-discharge adverse events. \\
\hline $\begin{array}{l}\text { Educating patients to promote } \\
\text { self-management }\end{array}$ & $\begin{array}{l}\text { Involves teaching patients and their caregivers about the main hospital diagnoses and instructions for } \\
\text { self-care, including medication changes, appointments, and whom to contact if issues arise. }\end{array}$ \\
\hline Coordinating care among team members & This is needed to synchronize efforts across settings and providers. \\
\hline $\begin{array}{l}\text { Monitoring and managing symptoms } \\
\text { after discharge }\end{array}$ & $\begin{array}{l}\text { Monitoring for new or worsening symptoms; medication side effects, discrepancies, or nonadherence; } \\
\text { and other self-management challenges will allow problems to be detected and addressed early, before } \\
\text { they result in unplanned healthcare utilization. }\end{array}$ \\
\hline Outpatient Follow-up & $\begin{array}{l}\text { Optimal follow-up with appropriate post-discharge providers is crucial for providing ideal transitions. } \\
\text { These appointments need to be prompt (e.g. within } 7 \text { days if not sooner for high-risk patients) and with } \\
\text { providers who have a longitudinal relationship to the patient, as prior work has shown increased } \\
\text { readmissions when the provider is unfamiliar with the patient }\end{array}$ \\
\hline
\end{tabular}


The Transition Nurse will provide facilitation using the Transition of Care Resource Guide and a Transition of Care Dashboard. This project was reviewed by the Colorado Multiple Institutional Review Board and deemed to be a QI project (See Fig. 2).

\section{Partnership approach}

Evaluation team Our multidisciplinary evaluation team consists of experts in the field of qualitative and quantitative research, Lean Six-Sigma, statistics and data management, implementation science, public health, nursing and medicine who bring together experience, knowledge, methodological rigor and skills for the success of this QI project.

Operational partners This project will be carried out in partnership with Office of Community Engagement, ECHCS stakeholders, VISN 19, Rural Health Resource Center-Western Region, the VA Office of Community Care as well as the Quality Enhancement Research Initiative (QUERI) Program which is providing protected time for all the personnel to participate in all aspects of this QI project. This partnership enables us to implement the proposed study design, evaluate the intervention's impact, and disseminate findings to other sites. The proposed Transition Nurse role is intended to augment current programs by ensuring care coordination for hospitalized Veterans returning to VA primary care.

Relationship between evaluation team and operational partners The multidisciplinary team has a longstanding presence in conducting Veteran-centered research with expertise in program evaluation, quality improvement initiatives and implementation research. The team is composed of individuals with experience providing scientific and methodological expertise, as well as QI efforts in their respective focus areas (medicine, public health, nursing, research). To ensure continued value and relevance of the project and engagement of key stakeholders with the project, a Strategic Advisory Group is in place to provide feedback, guidance and assistance for engagement with project sites throughout the project. The Strategic Advisory Group is composed of VA and non-VA leaders and key stakeholders.

\section{Key informant interviews}

We will conduct in-depth, semi-structured key-informant interviews pre-intervention development with VA and non-VA providers and staff informants as well as Veterans to understand the current transition of care, facilitators and barriers of the processes and obtain suggestions for improvement. The semi-structured interview guides will be aligned with domains from PRISM such as Program/ intervention-organizational perspective, Program/Intervention-Patient perspective, Implementation and Sustainability Infrastructure and Characteristics of Organizational recipients. Utilization of the PRISM model will assist in the assessment of the different organizational perspectives, characteristics of intervention recipients and translation of qualitative findings into a sustainable intervention. Participants will be recruited using convenience and snowball sampling from VA and non-VA hospitals frequented most by Veterans. Qualitative data analysis will be done using Atlas.ti software. Information obtained from the qualitative inquiry along with the results of Lean Six Sigma assessment will inform the development of the current transition process map and the value-stream map. Findings from the key informant interviews will help in designing an intervention to improve the existing transition process.

\section{Qualitative data analysis}

Utilize a conventional content analysis [19] technique for qualitative data analysis, interviews will be conducted

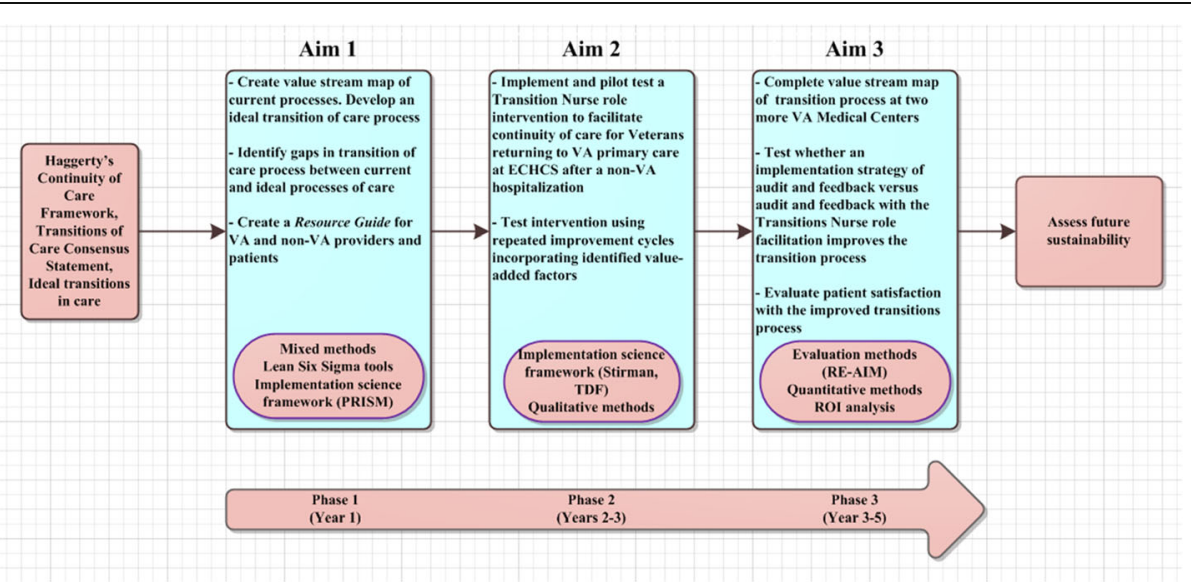

Fig. 2 Transitions of Care project description 
by evaluation team members and transcribed verbatim. Two analytic team members will independently code the initial interview for each role interviewed and meet to review the documents to clarify meanings of codes and come to consensus when disagreements occur, thus defining the initial code book. Emergent codes will be added to the code book as they come up [20-22]. Consensus building will be achieved by discussing coding consistency with the qualitative evaluation team and resolution of differences in coding among the evaluation team members. All transcripts will then be coded using Atlas.ti. This process will be repeated as interviews take place for each new site. The coded data will then be examined and organized into categories, domains, or themes. We will continue this process until no new concepts are identified. Results will be reviewed by members of the evaluation team to assess thoroughness and comprehensiveness [20,21].

\section{Analyses of Lean Six Sigma}

As part of the Lean Six Sigma approach, we will describe the components of the Define, Measure, and Analyze (DMAIC) assessment to analyze the current transition of care process. Initially, we will create a value stream map identifying current steps, documentation of time at each step, delays, and information flows required to deliver specific components of the transition process. This will help identify gaps in processes, target improvement priorities, and construct a roadmap to close gaps between ideal and current transition of care process map. Next, using the results of the qualitative data analysis, we will create a detailed process map of the steps Veterans take post discharge from a non-VA hospital to VA primary care. This will help visualize value added and non-value added activities in the current transition process.

Furthermore, we will utilize ideal process descriptions from key informant interviews and literature to map the ideal transition of care process. This will help visualize the gaps in transition of care process between the current and ideal process as well as prioritize targets for improvement. To conceptualize, develop and evaluate the intervention based on our understanding of the current transition process, we will identify activities, resources, and external factors that could influence the results. This will help us capture expected outcomes of the different intervention activities and ensure the intervention is aligned with the original intent of our study.

\section{Development of transition of care resource guide}

We will create a Transition of Care Resource Guide for VA and non-VA providers and Veterans to ensure implementation of the intervention. The Resource Guide will consist of two checklists: 1) provider checklist will identify physician name/contact information at non-VA hospital, discharge diagnosis/discharge plan, outstanding lab/imaging/pathology results as well as critical or high risk medication(s), and when the patient needs follow-up care. This checklist and medical records will be sent to VA primary care providers by non-VA hospitals; and 2) patient checklist that will provide information on followup care and key contact information at the VA. The resource guide items will allow for monitoring and feedback of the process during rollout in the next phase to ensure that pertinent information is received by VA primary care and that Veterans are receiving optimal continuity of care.

There are two major goals we want to accomplish in Aim 3. First we will implement and test the Transitions Nurse role in the field using repeated improvement cycles. Potential solutions will need to promote greater standardization to an otherwise fragmented delivery system. The different intervention elements of the Transitions Nurse role will be determined by what we learn about the current transition process from the qualitative interviews and Lean Six Sigma tools. We have successfully implemented a Transition Nurse role for Veterans who are referred from VA facilities/clinics to Denver VA for inpatient specialty care. Use of the Transition Nurse role has led to decrease in emergency department visits in the 30 days after hospital discharge with high provider and patient satisfaction [23].

Based on the review of the literature, some of the ways the Transition Nurse will facilitate implementation of the new processes include: 1) Collaborating with the non-VA discharge planners/case managers to complete needed documentation and/or medical record transfer. This provides VA primary care with essential details of the hospitalization and follow-up plan; 2) Obtaining follow-up appointment at the VA primary care within 14 days of discharge or sooner if clinically indicated; 3 ) Calling the Veteran within 72 hours after discharge to assess symptoms and concerns, perform medication reconciliation, verify planned follow-up appointment attendance, and assess discharge status (knowledge of selfcare, medications, whom to contact, and health literacy) of the patient [24]. The Transition Nurse will use teachback methodology to address gaps in discharge preparedness [25] and will remain available as a resource for the patient until the Veteran is reintegrated into VA primary care. If a Veteran does not have an assigned primary care provider, the Transition Nurse will facilitate getting a VA primary care assignment and follow-up.

The second goal of years $3-5$ is to conduct a value stream map of the transition process at two other VA Medical Centers in VISN 19. We will test whether an implementation strategy of audit and feedback (of the value stream map of the transition process) versus audit and feedback with nurse facilitation improves the transition process, continuity of care, satisfaction and clinical 
outcomes. We will scale up the evidence based intervention to two other VA medical centers. We will create a value stream map of their process, adapt the Transitions of Care Resource Guide to the local context of the Medical Centers and include site specific information. Then, we will work with each Medical Center to implement a Transition Nurse role or identify someone currently at the Medical Center who can fulfill the role. For all aims, we will use the Stirman Framework [26] to systematically track adaptations and modifications to both data gathering methods and the formation and adaptation of the intervention and its implementation.

\section{Evaluation of intervention}

We will evaluate our intervention and implementation strategy using the RE-AIM measures. RE-AIM measures are a common evaluation approach to assess the impact of the implementation strategies and sustainability.

Reach is defined in terms of the proportion of Veterans hospitalized at non-VA facility transitioning back to VA primary care. We will assess the absolute number, proportion, and representativeness of VA primary care, non-VA providers and patients who receive the Transition of Care Resource Guide checklist during the transition.

Effectiveness of the intervention will be evaluated by the following metrics: 1) adherence to Transition of Care Resource Guide on a monthly basis. We will set-up a collaborative teleconference with a plan for scheduled on-line meetings to allow stakeholders to talk through issues and problem solve as a group; 2) satisfaction of the patients' transition experience using the validated Care Transition Measure (CTM) [27, 28] and Likertscale questions to assess satisfaction, which will reflect the overall quality of transition process (8-12 calls/ month based on thematic saturation); 3) satisfaction of the providers (VA and non-VA) experience through key informant interviews; and 4) emergency department and re-hospitalizations in the 30 days after index hospital discharge from the non-VA hospital.

Adoption is the absolute number, proportion, and representativeness of settings and intervention agents (Medical Centers) who are willing to participate in the Transition Nurse program. Furthermore, adoption of the Transitions Nurse program internally at the Denver VA medical center and by participating patients will be evaluated.

Implementation is defined as the extent to which the intervention is implemented as intended as well as adaptations made to the intervention. We will focus on barriers and facilitators of the implementation and assess adaptations and modifications to the intervention using Stirman's framework [26]. We will also conduct a standardized Return on Investment analysis to assess intervention cost-benefit and future sustainability. Through the
Lean Six Sigma process, we will seek to understand how organizational context affects QI efforts, organizational context such as staffing, culture, teamwork, leadership, and communication $[29,30]$ have all influenced the uptake and sustainability of QI improvements. Because organizational context is important, we will collect data through interviews and field notes (during collaborative teleconferences) about job tasks, roles, and social dynamics. Additional details are in the implementation core section.

Maintenance: Maintenance will be assessed via longterm use of Transitions of Care Resource Guide and extent to which sites continue the Transition Nurse program to facilitate Veterans transition following hospital discharge. Furthermore, we will also evaluate the extent of patients continuing to participate in the Transitions Nurse program

\section{Discussion}

\section{Potential challenges and limitations}

This project aims to evaluate the current transition of care process and implement an intervention to promote smooth transition for Veterans hospitalized in a non-VA hospital and who return to VA for follow-up primary care. We will work with operational partners to identify deliverables viewed as having an important impact on improving the transition of care process for Veterans. These include: 1) Provide value stream map of processes that promotes continuity of care and create a Transition of Care Resource Guide with "ideal" transition processes and checklists; 2) An evaluation of the different evidence-based transitions of care approaches including the Transition Nurse will be added to the Transition of Care Resource Guide with templates for dashboards and interview guides, and 3) An Evaluation Report using the RE-AIM measures of the two implementation strategies to improve the transition process, continuity of care, and clinical outcomes following out of VA hospitalization will be completed.

A few limitations may arise during this project. First, different target audiences might have different reactions to using a Resource Guide which might not be updated regularly to accommodate the changing needs of different organizations (VA and non-VA). Another potential challenge lies with sustainability of a Transitions Nurse due to the cost associated with an additional FTE. The Return on Investment evaluation will provide valuable information about cost effectiveness of the intervention. Finally, developing an intervention to improve care transitions assumes that non-VA hospitals are able and willing to participate in improving the current process. This may limit the intervention adaption and outcomes of the intervention.

\section{Contribution to practice}

This project will contribute to the improvement of transitions of care processes for Veterans. As a result of 
the 2014 CHOICE Act, which provided eligible Veterans an opportunity to receive non-VA care, the number of Veterans receiving care in a non-VA hospital is increasing. Understanding the barriers and facilitators to the current transition of care process for these Veterans when they return to VA primary care is essential to ensure proper follow-up care, medication reconciliation, and transfer of information from non-VA hospital settings. Effective implementation of this project will help Veterans in one of the most vulnerable times post discharge from non-VA hospitalizations. This project is addressing an area that has not been evaluated in previous projects at ECHCS with input from interdisciplinary teams, local and national stakeholders.

\section{Abbreviations \\ ECHCS: Eastern Colorado Health Care System; PRISM: Practical, Robust Implementation and Sustainability Model; TDF: Theoretical domains framework; VA: Veterans Affairs; VA HSR\&D: Veterans Affairs Health Services Research and Development}

\section{Acknowledgements}

The study was made possible by funding from Veterans Affairs Health Services Research and Development grant. The qualitative data collection was done by the evaluation team members and VA claim data was pulled from nationally available VA corporate data warehouse. No sponsor had a role in the design and conduct of the study; collection, management, analysis, and interpretation of the data; and preparation, review, or approval of the manuscript. The authors would like to gratefully acknowledge study participants (Veterans, providers and staff) for sharing their time and perspectives.

\section{Funding}

This project was funded by Veterans Affairs Health Services Research and Development grant. The funding body had no role in the design of the study and collection, analysis, and interpretation of data and in writing the manuscript.

\section{Availability of data and material}

The datasets generated and/or analyzed during the current study are not publicly available due to identifying nature of patients and providers who participated in the qualitative interview. Furthermore, the VA claim data has patient data that is not to be shared publicly. However, how data was collected and managed can be shared including the interview guides via the corresponding author on reasonable request.

\section{Authors' contributions}

$\mathrm{CB}$ is the principal investigator and $\mathrm{BB}$ is a co-principal investigator for the project. CB was responsible for the study design, and methodological and analytic strategy. Methodological rigor support was provided by RG and BR for implementation science frameworks, RA, EL, MM, KF for qualitative methods and JP for project coordination plans. CB was responsible for the initial research question, securing and maintaining relationships with our operational partners, providing input, and materially edited this manuscript. RA had the responsibility of writing and coordinating with the team for this manuscript. RA, EL, MM, KF are research analysts on our study team who will carry out both qualitative and lean six sigma portions of the study protocol. All study team provided expertise in the methodology, materially edited this manuscript, will supervise study phases, and collaborate on data analysis. All authors read and approved the final manuscript.

\section{Competing interests}

The authors declare that they have no competing interests.

\section{Consent for publication}

Not applicable.

\section{Ethics approval and consent to participate}

Colorado Multiple Institutional Review Board (COMIRB) and Veterans Affairs Health Services Research and Development (VA HSR\&D) ethics review board deemed this a QI project on October 21, 2015.

\section{Author details}

'Department of Veterans Affairs, Eastern Colorado Health Care System, 1055 Clermont Street, Research (A151), Denver, CO 80220, USA. ${ }^{2}$ University of Colorado, Anschutz Medical Campus, Aurora, CO, USA. ${ }^{3}$ Department of Family Medicine and Public Health, School of Medicine, University of California San Diego, La Jolla, California, USA. ${ }^{4}$ Department of Family Medicine, School of Medicine, University of Colorado, Aurora, Colorado, USA.

Received: 13 December 2016 Accepted: 20 January 2017

Published online: 10 February 2017

\section{References}

1. Corbett CF, Setter SM, Daratha KB, Neumiller JJ, Wood LD. Nurse identified hospital to home medication discrepancies: implications for improving transitional care. Geriatr Nur (Lond). 2010;31(3):188-96.

2. Rehospitalizations among Patients in the Medicare Fee-for-Service Program NEJM [Internet]. [cited 2016 Aug 13]. Available from: http://www.nejm.org/doi/ full/10.1056/NeJmsa0803563\#t=article.

3. The Incidence and Severity of Adverse Events Affecting Patients after Discharge from the Hospital | Annals of Internal Medicine [Internet]. [cited 2016 Aug 13]. Available from: http://annals.org/article.aspx?articleid=716006.

4. Tsilimingras D, Bates DW. Addressing postdischarge adverse events: a neglected area. Jt Comm J Qual Patient Saf. 2008;34(2):85-97.

5. Bowles KH, Foust JB, Naylor MD. Hospital discharge referral decision making: a multidisciplinary perspective. Appl Nurs Res. 2003;16(3):134-43.

6. Improving Care Transitions [Internet]. Health Affairs - Health Policy Briefs. [cited 2016 Oct 14]. Available from: http://www.healthaffairs.org/healthpolicybriefs/ brief.php?brief_id=76.

7. Gittell JH, Fairfield KM, Bierbaum B, Head W, Jackson R, Kelly M, et al. Impact of relational coordination on quality of care, postoperative pain and functioning, and length of stay: a nine-hospital study of surgical patients. Med Care. 2000;38(8):807-19.

8. Kripalani S, LeFevre F, Phillips CO, Williams MV, Basaviah P, Baker DW. Deficits in communication and information transfer between hospital-based and primary care physicians: Implications for patient safety and continuity of care. JAMA. 2007;297(8):831-41.

9. Hynes DM, Koelling K, Stroupe K, Arnold N, Mallin K, Sohn M-W, et al. Veterans??? Access to and Use of Medicare and Veterans Affairs Health Care. Med Care. 2007:45(3):214-23.

10. Naylor MD, Aiken LH, Kurtzman ET, Olds DM, Hirschman KB. The importance of transitional care in achieving health reform. Health Aff (Millwood). 2011; 30(4):746-54.

11. Rother M, Shook J. Learning to see: value stream mapping to add value and eliminate muda. Cambridge: Lean Enterprise Institute; 2003. p. 115.

12. Feldstein AC, Glasgow RE. A practical, robust implementation and sustainability model (prism). Jt Comm J Qual Patient Saf. 2008;34(4):228-43.

13. Gaglio B, Shoup JA, Glasgow RE. The RE-AIM Framework: a systematic review of use over time. Am J Public Health. 2013;103(6):e38-46.

14. Haggerty JL, Reid RJ, Freeman GK, Starfield BH, Adair CE, McKendry R. Continuity of care: a multidisciplinary review. BMJ. 2003;327(7425):1219-21.

15. Snow V, Beck D, Budnitz T, Miller DC, Potter J, Wears RL, et al. Transitions of Care Consensus Policy Statement American College of Physicians-Society of General Internal Medicine-Society of Hospital Medicine-American Geriatrics Society-American College of Emergency Physicians-Society of Academic Emergency Medicine. J Gen Intern Med. 2009;24(8):971-6.

16. Burke RE, Kripalani S, Vasilevskis EE, Schnipper JL. Moving beyond readmission penalties: Creating an ideal process to improve transitional care. J Hosp Med. 2013;8(2):102-9.

17. Audit and feedback: effects on professional practice and health care outcomes - Jamtvedt - 2006 - The Cochrane Library - Wiley Online Library. [cited 2016 Oct 14]; Available from: http://onlinelibrary.wiley.com/doi/10. 1002/14651858.CD000259.pub2/abstract.

18. Hysong SJ. Meta-analysis: audit \& feedback features impact effectiveness on care quality. Med Care. 2009;47(3):356-63.

19. Hsieh H-F, Shannon SE. Three approaches to qualitative content analysis. Qual Health Res. 2005;15(9):1277-88. 
20. Creswell JW, Hanson WE, Plano VLC, Morales A. Qualitative research designs selection and implementation. Couns Psychol. 2007;35(2):236-64.

21. Curry LA, Nembhard IM, Bradley EH. Qualitative and mixed methods provide unique contributions to outcomes research. Circulation. 2009:1 19(10):1442-52.

22. Patton MQ. Qualitative research \& evaluation methods. SAGE; 2002. 692 p.

23. Coleman EA, Parry C, Chalmers S, Min S. The care transitions intervention: Results of a randomized controlled trial. Arch Intern Med. 2006;166(17):1822-8.

24. Chew LD, Griffin JM, Partin MR, Noorbaloochi S, Grill JP, Snyder A, et al. Validation of screening questions for limited health literacy in a large VA outpatient population. J Gen Intern Med. 2008;23(5):561-6.

25. Kornburger C, Gibson C, Sadowski S, Maletta K, Klingbeil C. Using "Teach-Back" to promote a safe transition from hospital to home: an evidence-based approach to improving the discharge process. J Pediatr Nurs. 2013;28(3):282-91.

26. Stirman SW, Miller CJ, Toder K, Calloway A. Development of a framework and coding system for modifications and adaptations of evidence-based interventions. Implement Sci. 2013;8:65.

27. Coleman EA, Mahoney E, Parry C. Assessing the quality of preparation for posthospital care from the patient's perspective: the care transitions measure. Med Care. 2005;43(3):246-55.

28. MD EAC, MPH, PhD CP, MSW, MA, MPH SAC, et al. The Central Role of Performance Measurement in Improving the Quality of Transitional Care. Home Health Care Serv Q. 2007; 28:26(4):93-104.

29. Glasgow JM, Yano EM, Kaboli PJ. Impacts of organizational context on quality improvement. Am J Med Qual Off J Am Coll Med Qual. 2013; 28(3):196-205

30. McCullough MB, Chou AF, Solomon JL, Petrakis BA, Kim B, Park AM, et al. The interplay of contextual elements in implementation: an ethnographic case study. BMC Health Serv Res. 2015;15(1):62.

\section{Submit your next manuscript to BioMed Central and we will help you at every step:}

- We accept pre-submission inquiries

- Our selector tool helps you to find the most relevant journal

- We provide round the clock customer support

- Convenient online submission

- Thorough peer review

- Inclusion in PubMed and all major indexing services

- Maximum visibility for your research

Submit your manuscript at www.biomedcentral.com/submit

C) Biomed Central 